Int. J. Electrochem. Sci., 12 (2017) $1572-1588$

Review

\title{
Recent Progress and Perspective Design of Various Electrode Catalysts for the Simultaneous Determinations of Dopamine in the Presence of Ascorbic Acid and Uric Acid: A Review
}

\author{
Rasu Ramachandran ${ }^{1}$, Tse-Wei Chen ${ }^{2,6}$, Shen-Ming Chen ${ }^{2, *}$, George peter Gnana kumar ${ }^{3}$, \\ Muthiah Chinnasamy ${ }^{4}$, Natrajan Biruntha Devi ${ }^{5}$, Tien-Wen Tseng ${ }^{6}$ \\ ${ }^{1}$ Department of Chemistry, The Madura College, Vidya Nagar, Madurai - 625 011, Tamil Nadu, India. \\ ${ }^{2}$ Electroanalysis and Bioelectrochemistry Lab, Department of Chemical Engineering and \\ Biotechnology, National Taipei University of Technology, No.1, Section 3, Chung-Hsiao East Road, \\ Taipei 106.Taiwan (ROC). \\ ${ }^{3}$ Department of Physical Chemistry, School of Chemistry, Madurai Kamaraj University, Madurai - 625 \\ 021, Tamil Nadu, India. \\ ${ }^{4}$ Department of Inorganic Chemistry, School of Chemistry, Madurai Kamaraj University, Madurai - \\ 625 021, Tamil Nadu, India. \\ ${ }^{5}$ Department of Chemsitry, S.Vellaichamy Nadar College, Nagamalai pudukkottai, Madurai - 625021, \\ Tamil Nadu, India. \\ ${ }^{6}$ Department of Chemical Engineering and Biotechnology, National Taipei University of Technology, \\ No.1, Section 3, Chung-Hsiao East Road, Taipei 106.Taiwan (ROC). \\ *E-mail: smchen78@ms15.hinet.net
}

doi: $10.20964 / 2017.02 .76$

Received: 24 November 2016 / Accepted: 23 December 2016 / Published: 30 December 2016

The flexible electrochemical based biosensors have been fabricated by various suggested methods and the designed composite offer new possibilities for the evaluation of biosensor applications. The resulting composite brought new kind of capabilities, which were employed electrochemical performance and greatly enhanced electrocatalytic activities of DA, AA and UA. The reviewed electrode catalysts have extensively been used improved the electrocatalytic activity and quantitative estimation of real sample analysis from human urine and blood serum. We have briefly discussed different parameters on the basis of biosensors, such as sensitivity, selectivity, reproducibility, real sample analysis and electrode stability. This article offers useful way, which could expand low-cost electrode (disposable electrode) materials and hold great promise electrochemical biosensing devices for biosensor applications.

Keywords: Electrode catalysts, Biosensors, Dopamine, Ascorbic acid, Uric acid, Real sample, Electrode stability. 


\section{FULL TEXT}

(C) 2017 The Authors. Published by ESG (www.electrochemsci.org). This article is an open access article distributed under the terms and conditions of the Creative Commons Attribution license (http://creativecommons.org/licenses/by/4.0/). 